\title{
The Trophic Role of Oligodendrocytes in the Basal Forebrain
}

\author{
Xudong Dai, ${ }^{1}$ Lauren D. Lercher, ${ }^{1}$ Patricia M. Clinton, ${ }^{1}$ Yangzhou Du, ${ }^{1}$ Denise L. Livingston, ${ }^{1}$ Cristina Vieira, ${ }^{1}$ Lu Yang, ${ }^{2}$ \\ Michael M. Shen, ${ }^{2}$ and Cheryl F. Dreyfus ${ }^{1}$ \\ ${ }^{1}$ Department of Neuroscience and Cell Biology and ${ }^{2}$ Center for Advanced Biotechnology and Medicine and Department of Pediatrics, University of Medicine \\ and Dentistry of New Jersey-Robert Wood Johnson Medical School, Piscataway, New Jersey 08854
}

Traditionally, the primary function of oligodendrocytes (OLGs) in the CNS has been considered to be myelination. Here, we investigated whether OLGs may play a trophic role, particularly during development. Neurotrophin expression was assessed in postnatal day 7 basal forebrain (BF) OLGs, using in situ hybridization and detection of myelin basic protein. Nerve growth factor, brain-derived neurotrophic factor (BDNF) and neurotrophin-3 (NT-3) mRNAs were revealed in OLGs in vivo and in culture. To determine whether OLGs support nearby neurons, we examined the influence of OLGs on BF cholinergic neurons. Neuronal function was enhanced by cocultured OLGs and OLG conditioned medium. Moreover, trophic effects of OLG conditioned medium were partially blocked by K252a, a trk tyrosine kinase inhibitor, and by neutralizing anti-BDNF or anti-NT-3 antisera, indicating that neurotrophins may mediate these effects, perhaps in concert with other signals. Our studies support a novel role for OLGs in providing local trophic support for neurons in the CNS.

Key words: oligodendrocytes; basal forebrain; NGF; BDNF; NT-3; trophic role of oligodendrocytes on cholinergic neurons

\section{Introduction}

Although oligodendrocytes (OLGs) traditionally have been defined as myelin providers and maintainers (Szuchet, 1995) in the CNS, a variety of recent reports suggest that these cells play additional roles. For example, OLGs in culture express a variety of trophic molecules (Raabe et al., 1997; Strelau and Unsicker, 1999; Wilkins et al., 2001). However, direct evidence in general that OLGs express these molecules in vivo and use these molecules to support nearby neurons has been lacking (but see Nakamura et al., 1999).

To address this issue, we examined the expression of neurotrophins in basal forebrain (BF) OLGs in vivo and in culture, and have examined the trophic influences of BF OLGs on local cholinergic neurons. These BF cholinergic neurons are known to be highly responsive to the following neurotrophins: nerve growth factor (NGF), brain-derived neurotrophic factor (BDNF), and neurotrophin-3 (NT-3). In particular, NGF enhances the function of embryonic cholinergic neurons by increasing activity of the acetylcholine-synthesizing enzyme choline acetyltransferase (ChAT) (Gnahn et al., 1983; Martinez et al., 1985; Mobley et al., 1986; Knusel et al., 1990), elevating acetylcholine in postnatal day 1 (P1) septal neurons (Takei et al., 1988), and supporting survival of adult cholinergic cells (Hefti, 1986), particularly after a lesion. BDNF and NT-3 have been shown to increase the number of the acetylcholinesterase (AChE)-positive cholinergic neurons in culture (Alderson et al., 1990; Friedman et al., 1993). In addition, the

Received 0ct. 22, 2002; revised April 24, 2003; accepted April 28, 2003.

This work was supported by National Institutes of Health Grants HL60212, HD38766 (M.M.S.), and NS36647 (C.F.D.) and by the National Multiple Sclerosis Society (C.F.D.). We thank Drs. Wilma Friedman and lra Black for their critical and helpful discussion of this work.

Correspondence should be addressed to Dr. Cheryl F. Dreyfus, Department of Neuroscience and Cell Biology, University of Medicine and Dentistry of New Jersey-Robert Wood Johnson Medical School, 679 Hoes Lane, Piscataway, NJ 08854. E-mail: dreyfus@cabm.rutgers.edu.

Copyright $\odot 2003$ Society for Neuroscience $\quad$ 0270-6474/03/235846-08\$15.00/0 p75 and trk tyrosine kinase neurotrophin receptors have been found to be present on cholinergic neurons.

We used this system to address the following three questions: (1) Do BF OLGs express neurotrophins in vivo and in culture? (2) What biological influence do OLGs have on neurotrophinresponsive neurons in the $\mathrm{BF}$ ? (3) Are neurotrophins involved in OLG-neuron trophic interactions? Our study has found that NGF, BDNF, and NT-3 are produced by OLGs in vivo and in culture and demonstrates trophic effects of these molecules on nearby neurons. Together, our results support a trophic interaction between OLGs and nearby neurons in the CNS.

Preliminary findings of this work have been published previously in abstract form (Dai et al., 1997, 1998).

\section{Materials and Methods}

Experimental animals. Sprague Dawley rats were obtained from Hilltop Laboratories (Scottdale, PA) and housed in clear plastic cages. Food and water were available ad libitum. The day of birth was considered to be P0. The animals were managed by the UMDNJ-Robert Wood Johnson Animal Facility, which is accredited by the Association for Assessment and Accreditation of Laboratory Animal Care. Animal maintenance, transportation, and housing were in compliance with the Laboratory Animal Welfare Act (PL-89-544; PL-91-579). Moreover, our use of animals is in compliance with National Institutes of Health $(\mathrm{NIH})$ guidelines $(\mathrm{NIH}$ manual, chapter 4206).

Oligodendrocyte-enriched cultures. To prepare OLG cultures, we used a modification (O'Malley et al., 1991) of the method of McCarthy and DeVellis (1980). Rat BFs were dissected from P1 time-mated Sprague Dawley rats and cultured in nutrient medium-15 (NM-15) medium containing Minimum Essential Medium with Earle's salts and L-glutamine, heat-inactivated fetal calf serum $(15 \%)$, glucose $(6 \mathrm{mg} / \mathrm{ml})$, and penicillin-streptomycin $(0.5 \mathrm{U} / \mathrm{ml}$ and $0.5 \mu \mathrm{g} / \mathrm{ml}$, respectively) (Invitrogen, Rockville, MD) for 7-10 d. To eliminate microglia, glial cultures were shaken at $425 \mathrm{rpm}$ for $10 \mathrm{~min}$, and the supernatant was discarded. After recovery in NM-15, the oligodendrocyte type-2-astrocyte progenitor cells and OLGs were separated from underlying astrocytes by shaking at 
$250 \mathrm{rpm}$ for $12-16 \mathrm{hr}$. To further remove microglia, the supernatant was plated onto uncoated $100 \mathrm{~mm}$ plastic dishes for $1 \mathrm{hr}$ at room temperature. OLG lineage cells were plated onto $35 \mathrm{~mm}$ poly-D-lysine-precoated dishes for immunocytochemical study or onto aminoalkysilaneprecoated slides (Sigma, St. Louis, MO) for the in situ hybridization study in NM-15 medium at a density of $3 \times 10^{4}$ cells $/ \mathrm{cm}^{2}$. After $24 \mathrm{hr}$ in OLG medium (OM), NM-15 medium was aspirated and replaced with chemically defined medium consisting of a 1:1 mixture of Ham's F12 and Basal Medium Eagle containing $6 \mathrm{mg} / \mathrm{ml}$ glucose and $6.6 \mathrm{~mm}$ glutamine (Invitrogen) and $25 \mu \mathrm{g} / \mathrm{ml}$ insulin, $20 \mathrm{~nm}$ progesterone, $60 \mu \mathrm{M}$ putrescine, $30 \mathrm{~nm}$ selenium, $100 \mu \mathrm{g} / \mathrm{ml}$ transferrin, $0.5 \mu \mathrm{M}$ thyroxin, $0.08 \mu \mathrm{g} / \mathrm{ml}$ triiodothyronine, $6.6 \mathrm{~mm}$ glutamine, and penicillin-streptomycin $(0.5$ $\mathrm{U} / \mathrm{ml}$ and $0.5 \mu \mathrm{g} / \mathrm{ml}$, respectively) (Sigma). Cultures were maintained for $96 \mathrm{hr}$ in OM, after which $>90 \%$ of the cells in culture were OLG lineage cells, either A2B5-positive or myelin basic protein (MBP)-positive immunocytochemically. The remaining cells were OX-42-positive or ED1-positive microglia or glial fibrillary acidic protein-positive (GFAP) type 1 astrocytes.

In some cases, we further enriched the OLG cultures. Two approaches were taken. First, after the shaking procedure, cells were plated on plastic dishes for $3 \mathrm{hr}$ to remove microglia and then treated with the microglial toxin L-leucine methyl ester (10 mM, 1 hr; Sigma) (Guillemin et al., 1997) and/or the astrocyte toxin D- $\alpha$-amino adipic acid (600 $\mu \mathrm{M}$; Sigma) (O'Malley et al., 1994). Optimal concentrations were determined experimentally. In the case of L-leucine methyl ester, cultures were then transferred to NM-15 and grown in the absence of toxin. In the case of $\alpha$-amino adipic acid, cultures were exposed to drug for the entire culture period.

In a second approach, cells were immunopanned according to previous methods (Plant et al., 2002). In brief, cells were plated on $100 \mathrm{~mm}$ Petri dishes that had been sequentially treated with biotinylated antimouse IgG (1:100, $12 \mathrm{hr}, 4^{\circ} \mathrm{C}$; Vector Laboratories, Burlingame, CA), A2B5 antibody with $5 \%$ BSA $\left(12 \mathrm{hr}, 4^{\circ} \mathrm{C}\right)$, and $5 \%$ BSA ( $4 \mathrm{hr}$, room temperature; Sigma). Cells were incubated in these dishes for $30 \mathrm{~min}$ at $37^{\circ} \mathrm{C}$. After three culture washes in NM-15, bound cells were scraped, centrifuged (at $500 \mathrm{rpm}$ for $5 \mathrm{~min}$ ), resuspended in NM-15, and plated.

Dissociated neuronal cultures. For neuronal culture studies, dissociated embryonic day 17 (E17) rat basal forebrains were plated on $35 \mathrm{~mm}$ polyD-lysine-coated plastic plates at $1 \times 10^{5} \mathrm{cells} / \mathrm{cm}^{2}$. Neurons were cultured in either OM (control group) or $50 \%$ OLG conditioned medium $(\mathrm{CM}) / 50 \% \mathrm{OM}$ (treatment group) for 2-7 $\mathrm{d}$ and then subjected to assay.

OLG-neuron coculture. To assess the influence of OLGs on neurons, OLG-neuron cocultures were established. The newly enriched P1 OLGs and their progenitors were added to the neuronal cultures immediately after neurons were plated. Cocultures were maintained for 5-7 d before additional assay.

OLG conditioned medium. To obtain OLG CM, enriched OLGs were grown in OM for $96 \mathrm{hr}$.

Nonradioactive in situ hybridization. For in vivo studies, coronal sections were obtained from P7 rats and mounted on aminoalkysilaneprecoated slides. Neurotrophin mRNAs were detected using digoxigenin-labeled riboprobes (Sciavolino et al., 1997; Yang et al., 1998). Sense and antisense riboprobes were derived from an NGF plasmid (kindly provided by Dr. Scott Whittemore, University of Miami School of Medicine, Miami, FL), a BDNF plasmid (kindly provided by Dr. Dale Schaar, UMDNJ-Robert Wood Johnson Medical School), and an NT-3 plasmid (kindly provided by Dr. Wilma J. Friedman, Rutgers University, Newark, NJ). These probes were labeled by digoxigenin-11UTP using a riboprobe in vitro transcription system (Promega, Madison, WI). To label OLGs after nonradioactive in situ hybridization in vivo, the $\mathrm{DAB}$ procedure was performed as described below for visualization of MBP. To label OLGs in culture after nonradioactive in situ hybridization, samples were visualized for MBP using immunofluorescence techniques. To estimate numbers of MBP-positive cells that expressed NGF, BDNF, or NT-3 mRNAs, three coronal sections from the BF were evaluated for each neurotrophin. Within each section, MBP-positive cells that expressed a particular neurotrophin were counted and compared with the total numbers of MBP-positive OLGs.

Immunostaining. Cell-specific markers were used to characterize
OLG-enriched cultures. These included polyclonal antisera against MBP (kindly provided by Dr. David Colman, Montreal Neurological Institute, Montreal, Quebec, Canada) (1:1000), monoclonal antibody A2B5 (1: 1000 ) derived from mouse lymphocyte hybridoma cells (American Type Culture Collection, Manassas, VA), polyclonal antisera against GFAP (1:2000; Dako, Carpinteria, CA), and monoclonal antibodies against OX-42 (1:1000; Serotec, Raleigh, NC) and against ED-1 (1:500; Serotec). Controls consisted of processing the tissues in the absence of primary antisera or antibodies. Optimal dilutions of these antisera or antibodies were determined experimentally. Neurotrophins were detected with polyclonal antisera to BDNF (1:500), NT-3 (1:1000), or NT-4 (1:1000), developed and kindly provided by David Kaplan (The Hospital for Sick Children, Toronto, Ontario, Canada). The specificity of these antisera has been confirmed in previous work (Friedman et al., 1998; Dreyfus et al., 1999). As negative controls, BDNF, NT-3, and NT-4 antisera were preadsorbed with appropriate purified BDNF, NT-3, and NT-4 $(1 \mu \mathrm{g} / \mathrm{ml}$; PeproTech, Rocky Hill, NJ); in some cases, preimmune rabbit serum was used. Samples were either exposed to primary antisera and then incubated in biotinylated anti-rabbit antiserum (1:500, 60 min; Vector Laboratories) or exposed to monoclonal antibodies and incubated in biotinylated anti-mouse antiserum followed by detection using the $\mathrm{ABC}$ technique (Vector Laboratories). No staining was observed with preadsorbed antibodies or control serum, consistent with previous observations (Friedman et al., 1998; Dreyfus et al., 1999). For immunofluorescence staining after exposure to MBP primary antiserum, cultures were incubated with FITC-conjugated goat anti-rabbit serum (Vector Laboratories) and were examined with a Leitz Aristoplan fluorescence microscope equipped with filter cube L3 for FITC fluorescence $(450-490 \mathrm{~nm}$ excitation filter). For cell counts, $3-6 \%$ of the dish was counted.

ChAT activity. ChAT was measured by a modification of the Fonnun procedure (Fonnun, 1975).

Histochemical staining for acetylcholinesterase. Plates were stained for acetylcholinesterase-positive neurons by the method of Geneser-Jensen and Blackstad (1971), as modified (Hefti et al., 1985). All positively stained cells across the diameter of each culture dish were counted to define the number of AChE-positive cells in the dish. The area examined represented $3 \%$ of the total area per dish.

Colabeling for acetylcholinesterase and bromodeoxyuridine. When DNA synthesis was assessed in cholinergic neurons, cultures were exposed to CM or serum-free medium (SFM) for 2, 4 , or $7 \mathrm{~d}$ and treated with bromodeoxyuridine (BrdU) for the last $4 \mathrm{hr}$ of each of these treatment periods. In one group of cultures, dishes were treated with BrdU from days 1 to 4 and then fixed. Cultures were stained first for AChE, as above, and then for BrdU using the methods of DiCicco-Bloom et al. (1993). Cultures were exposed to anti-BrdU (BD, San Diego, CA) and visualized with DAB (Sigma). The area examined represented $6 \%$ of the total area of the dish.

Colabeling for AChE and terminal deoxynucleotidyl transferasemediated biotinylated UTP nick end labeling. When cell death was assessed in cholinergic neurons, cultures were exposed to CM or SFM for 2, 4, or $7 \mathrm{~d}$. They were first stained for AChE and then for terminal deoxynucleotidyl transferase-mediated biotinylated UTP nick end labeling (TUNEL), a marker of cell death, according to the manufacturer's instructions (Roche Applied Science, Indianapolis, IN). Approximately 10\% of the dish was counted.

Neutralizing antisera for neurotrophins. Both anti-BDNF and antiNT-3 neutralizing antibodies (Promega) were raised against full-length human recombinant BDNF and NT-3, respectively. Anti-NGF antibody (Sigma) was raised to the $2.5 \mathrm{~S}$ form of NGF. These antibodies have been reported to neutralize neurotrophin action in other systems (Mazzoni and Kenigsberg, 1997; Toma and Kaplan, 1997; Meier et al., 1999). The anti-NT-3 does not cross-react with other members of neurotrophin family using dot blot or Western blot analysis (Promega and our unpublished observation). Anti-BDNF is reported to have cross-reactivity of $<3 \%$ with other neurotrophins as revealed by ELISA (Promega) and does not cross-react with other members of the neurotrophin family as demonstrated by Western blot analysis (our unpublished observations). Anti-NGF does not cross-react with other members of the neurotrophin family using Western blot analysis (our unpublished observation). Fur- 
thermore, these antibodies completely abolished increases in ChAT activity induced by authentic NGF, BDNF, or NT-3 in our cultures.

To neutralize NGF, BDNF, or NT-3, solutions containing authentic neurotrophins or $\mathrm{CM}$ solutions were preadsorbed at $4^{\circ} \mathrm{C}$ for $2 \mathrm{hr}$ with appropriate neutralizing antibodies and then exposed to the cultures. Control groups received control IgG (R \& D Systems, Minneapolis, MN) (anti-NGF) or IgY (Promega) (anti-BDNF or anti-NT-3).

It should be noted that not all batches of CM were affected equally by antibody treatment. Lack of blockade was observed in one-seventh of culture experiments for both anti-BDNF and anti NT-3, suggesting that levels of BDNF and NT-3 may vary between CM batches. Alternatively, because not all oligodendrocytes express BDNF or NT-3, the contribution of individual neurotrophins or of other trophic molecules to the effects of CM may correlate with the dominant oligodendrocyte subtype in our cultures.

\section{Results \\ Expression of NGF, BDNF, and NT-3 mRNA in vivo}

To determine whether BF OLGs express neurotrophins, we used a nonradioactive in situ hybridization method combined with immunocytochemical detection of the OLG marker MBP. In coronal forebrain sections from $\mathrm{P7}$ rats, MBP was colocalized with NGF (Fig. 1A), BDNF (Fig. 1B), or NT-3 (Fig. 1C) mRNAs. MBP-positive cells were either large, morphologically complex cells (25-40 $\mu \mathrm{m}$ diameter) or small cells (10-15 $\mu \mathrm{m})$ with few processes (Fig. 1). NGF, BDNF, and NT-3 mRNAs were observed in both cell types. Specific expression of mRNA for each neurotrophin was detected primarily in cell bodies; no signal was observed when sense riboprobes were used (data not shown). Interestingly, in no case was an individual neurotrophin expressed in all MBP-positive OLGs. In representative sections, $50 \%$ of the MBP-positive cells were positive for NGF mRNA, $39 \%$ of the MBP-positive cells were positive for BDNF mRNA, and $64 \%$ of the MBP-positive cells were positive for NT-3 mRNA, suggesting that subgroups of OLGs express individual neurotrophins in vivo.

To determine whether OLGs in other forebrain regions also express neurotrophins, corpus callosum and frontal, cingulate, and parietal cortical regions were examined. Interestingly, NGF, BDNF, and NT-3 mRNAs were observed in MBP-positive OLGs from all of these areas (Fig. 2). The relatively wide distribution of neurotrophin-expressing OLGs in the forebrain suggests that provision of neurotrophins may be a function of OLGs throughout the developing forebrain.

\section{Expression of neurotrophins in OLGs in culture}

To explore trophic effects of BF OLGs on nearby neurons, enriched OLG cultures were established from P1 BF. NGF, BDNF, and NT-3 mRNAs, revealed by in situ hybridization (Fig. $3 B, F, H)$, were detected in MBP-positive cells identified by im-
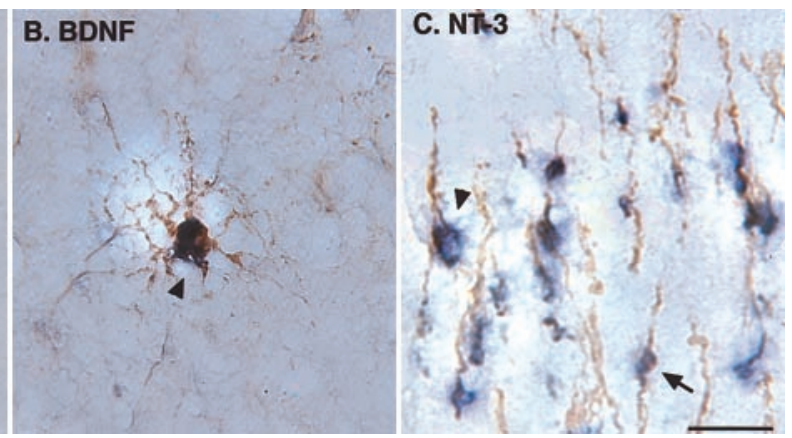

Figure 1. Expression of neurotrophins by MBP-positive OLGs in the P7 BF. Nonradioactive in situ hybridization reveals that NGF, DNF, and NT-3 mRNA are coexpressed with MBP in vivo. Coronal sections were prepared from P7 rats and examined by in situ DAB precipitation, is colocalized with $\operatorname{NGF}(A), \operatorname{BDNF}(B)$, or NT-3 $(C)$ mRNA, visualized by purple precipitation. Scale bar, $50 \mu \mathrm{m}$.
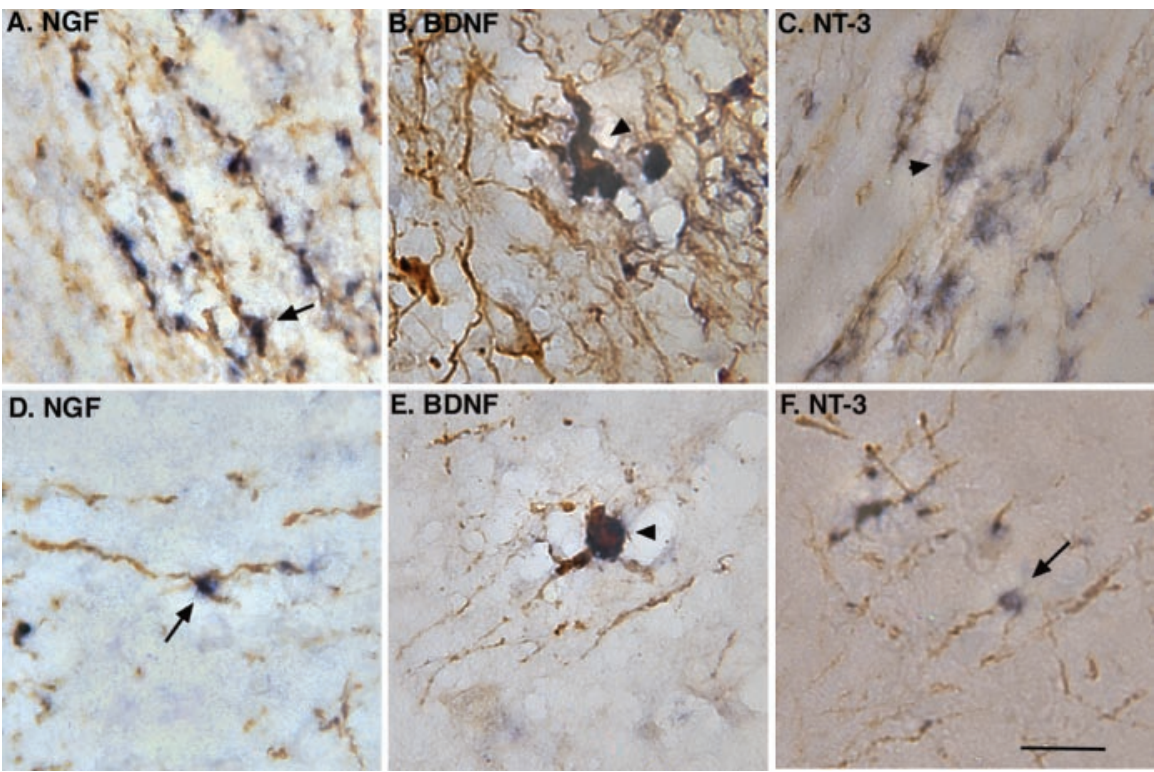

Figure 2. Expression of neurotrophins by MBP-positive OLGs in other forebrain structures. In the corpus callosum, MBP posiivity, visualized by brown DAB precipitation, is colocalized with NGF $(A)$, BDNF $(B)$, or NT-3 (C) mRNA, visualized by purple precipitation. In the frontal cortex, some MBP-positive cells also express NGF $(D), \operatorname{BDNF}(E)$, and NT-3 ( $F)$ mRNA. Such labeling was also observed in parietal and cingulate cortex. Scale bar, $50 \mu \mathrm{m}$. Arrows indicate small cells. Arrowheads indicate large cells.

munocytochemical staining (Fig. $3 A, G$ ). As was the case in vivo, in no instance was an individual neurotrophin detected in all multipolar OLGs (arrowheads).

To determine whether the mRNA in OLGs was translated into neurotrophin protein, immunocytochemical methods were used. Immunocytochemical staining for BDNF, NT-3, or NT-4 revealed that neurotrophins were present in multipolar OLG-like cells in enriched OLG cultures (Fig. $4 A-C$ ). The specificity of the staining was confirmed by using preimmune serum (Fig. $4 D$ ) and by antiserum preadsorbed with excess amounts of BDNF, NT-3, or NT-4 (data not shown).

Interestingly, we observed that neurotrophin protein immunoreactivity was present in virtually every OLG-like cell in culture, in contrast to the more heterogeneous mRNA expression pattern. One possible explanation for this finding is that neurotrophin-expressing OLGs release neurotrophins into the medium, and other OLGs that do not express neurotrophins themselves may take up neurotrophins in culture. In other studies, we found that BF OLGs express the common neurotrophin receptor $\mathrm{p} 75$, as well as trkA, trkB, and trkC 

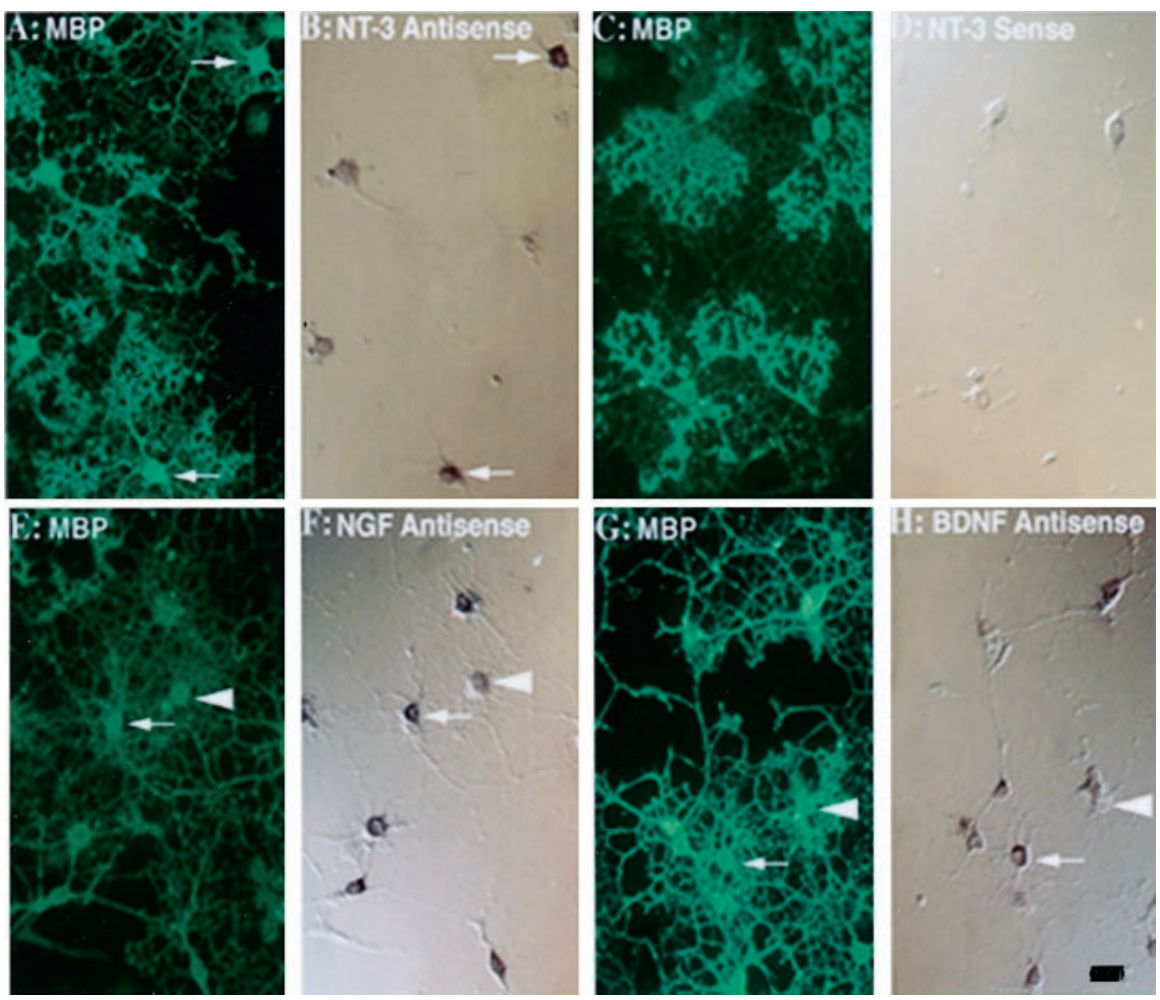

Figure 3. Expression of neurotrophin mRNAs by MBP-positive cells in culture. MBP-positive cells, visualized by FITC-conjugated horse anti-rabbit antibody $(A, E, G)$ express mRNAs for $\operatorname{NGF}(F), \operatorname{BDNF}(H)$, or NT-3 $(B)$, revealed by digoxigenin-11-UTP-labeled riboprobes and visualized as purple precipitation (arrows). Not all MBP-positive cells are positive for neurotrophin (arrowheads). The sense probes for the neurotrophins served as negative controls $(D)$. Similar patterns were observed in other controls (data not shown). Scale bar, $50 \mu \mathrm{m}$.
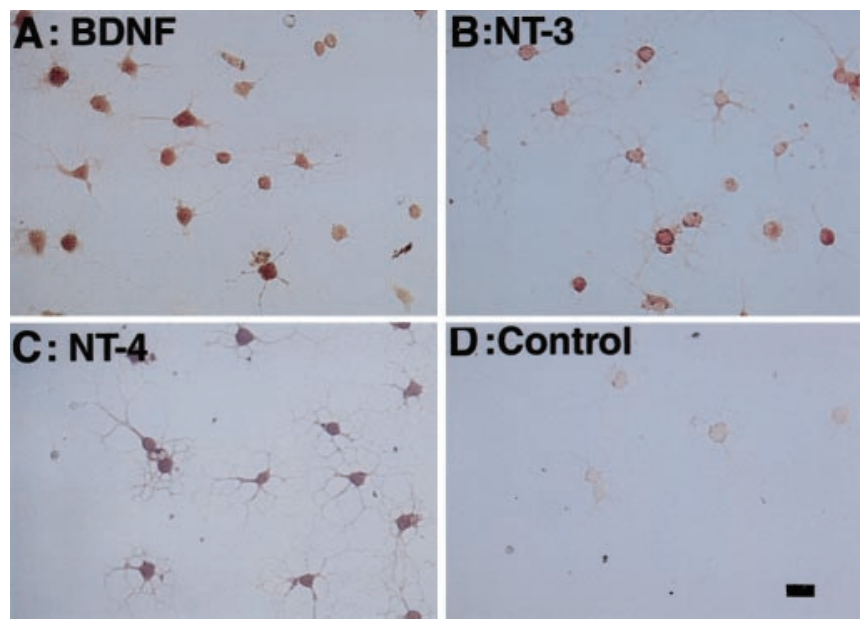

Figure 4. Expression of neurotrophin protein in OLG-like cells. P1 OLG-enriched cultures were immunocytochemically stained by primary antisera against $\operatorname{BDNF}(A)$, NT-3 $(B)$, or NT-4 (C). BDNF, NT-3, and NT-4 immunoreactivity is detected on most of the oligodendrocyte-like cells. In negative controls, preimmune serum $(D)$ or antibodies preadsorbed with appropriate neurotrophins reveal no staining. Scale bar, $50 \mu \mathrm{m}$.

receptors (Du et al., 2000), supporting this possibility. Alternatively, there may be different detection sensitivities for the in situ and immunocytochemical assays.

\section{Trophic effects of OLGs on BF cholinergic neurons} in coculture

The expression of neurotrophins in BF OLGs suggests that OLGs may provide trophic support to nearby neurons. To assess this potential trophic function, we cocultured OLGs with BF neurons. E17 BFs were dissociated and plated in chemically defined medium. Previous studies have indicated that this method results in $>95 \%$ neuronal cultures (Yokoyama et al., 1994). Neurons were cultured with or without enriched P1 OLGs for $5 \mathrm{~d}$. Coculture of OLGs with BF neurons elicited a fourfold increase in ChAT activity compared with neurons grown alone (Fig. 5A), whereas OLGs cultured by themselves as a control did not express ChAT activity. This result indicates that OLGs express molecules that can provide trophic influences to $\mathrm{BF}$ cholinergic neurons.

\section{Trophic effects of OLG CM on BF neurons in culture}

To determine whether diffusible factors such as neurotrophins might mediate the trophic influence of OLGs, we examined the effects of OLG CM on BF cholinergic neurons. BF neurons were cultured in the presence or absence of OLG CM, and ChAT activity was measured (Fig. 5B). $\mathrm{CM}$ treatment approximately tripled ChAT activity compared with the control, suggesting that molecules released by the OLGs directly affect cholinergic neurons in culture.

To determine whether the CM may affect other aspects of cholinergic neuron function, effects on the number of AChE-positive cells were investigated (Fig. $5 C$ ). CM treatment elicited a fourfold increase in the number of AChE-positive cells. Because AChE is expressed by basal forebrain cholinergic neurons (Eckenstein and Soforniew, 1983), these data suggest that CM increases the number of cholinergic neurons in culture.

To define the mechanisms underlying the increase in cholinergic neuron number in the CM-treated cultures, we examined effects of $\mathrm{CM}$ on BrdU incorporation, an index of DNA synthesis, or on TUNEL staining, an index of cell death. When cultures were exposed to $\mathrm{CM}$ or OM for 2, 4, or $7 \mathrm{~d}$, no AChE-positive cells were found to be BrdU-positive, although other cells in the dish did exhibit BrdU positivity. The data suggest that cholinergic neurons do not divide in these cultures, and CM does not affect this observation (data not shown). However, when TUNEL-positive, AChE-positive cholinergic neurons were evaluated, there was a marked decrease in the proportion of cholinergic neurons that exhibited TUNEL in the CM group, suggesting that CM decreases cell death (Fig. 5D). This was true whether cultures were evaluated on day 2,4 , or 7 .

\section{Trophic effects of BF OLGs are partially mediated by neurotrophins}

To determine whether the effects of CM are mediated through neurotrophins, we took two approaches. First, we examined the effects of K252a (Knusel and Hefti, 1992) on the activity of CM. In this experiment, neuronal cultures were exposed to CM plus DMSO vehicle or CM plus K252a (100 nM) for $5 \mathrm{~d}$, and were compared with cultures grown in normal OLG medium (control). We found that K252a partially inhibited the effect of CM on ChAT activity (Fig. 6A) but did not affect control levels of ChAT. 

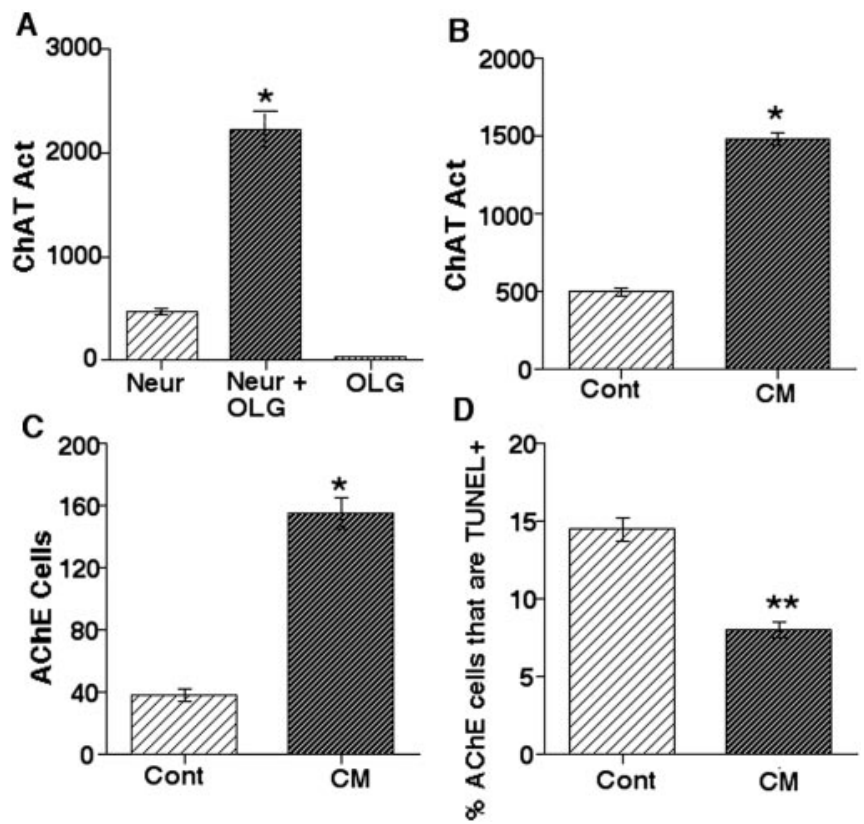

Figure 5. Trophic effects of OLGs on BF cholinergic neurons. $A$, Coculturing $0 L$ Gs with E17 BF neurons for $5 \mathrm{~d}$ increases the activity (Act) of ChAT in culture. B, C, BF OLG CM exposure for $5 \mathrm{~d}$ increases both ChAT activity $(B)$ and the number of $A C h E-$ positive cells $(C)$. CM exposure for $7 \mathrm{~d}$ decreases the percentage of AChE-positive cells that are TUNEL positive $(D)$. One of three $(A-C)$ or two $(D)$ independent experiments that yielded similar results are shown. Sample sizes for experiments: $n=15(A) ; n=10(B-D)$ (5 dishes per group). Neur, Neuron; Cont, control. *Significantly different from control at $p<0.05$. Data were analyzed by Student's $t$ test $(B-D)$ or ANOVA and the Scheffe's test $(A)$. ${ }^{*}$ Significantly different from control at $p<0.01$.

Second, we used neutralizing antibodies against individual neurotrophins. We used neutralizing antibodies that have been reported to specifically block the actions of BDNF, NGF, or NT-3 in other systems (Mazzoni and Kenigsberg, 1997; Toma and Kaplan, 1997; Meier et al., 1999), without cross-reactivity against other neurotrophins (see Materials and Methods). To determine whether BDNF is a component of the activity of CM, cultures were maintained for $6 \mathrm{~d}$ in $\mathrm{CM}$ or CM preadsorbed with neutralizing antiserum developed against full-length BDNF. To confirm that anti-BDNF neutralizes the effects of BDNF, in the same experiment, BDNF and BDNF preadsorbed with anti-BDNF were included (Fig. $6 \mathrm{~B})$. Anti-BDNF $(10 \mu \mathrm{g} / \mathrm{ml})$ completely blocked the effects of authentic BDNF $(10 \mathrm{ng} / \mathrm{ml})$ on ChAT activity in cultures. (This concentration of anti-BDNF was shown in our other studies to block BDNF at $50 \mathrm{ng} / \mathrm{ml}$.) When the identical concentration of anti-BDNF was applied to CM, we found that the antiserum partially blocked the effects of CM. Similar findings were obtained using anti-NT-3 $(10 \mu \mathrm{g} / \mathrm{ml})$ (Fig. $6 C)$. In contrast to anti-BDNF and anti-NT-3, neutralizing anti-NGF antiserum $(0.1 \mu \mathrm{g} / \mathrm{ml})$ failed to block the effects of CM on ChAT activity in any experiment (Fig. 6D). These data indicate that BDNF and NT-3 derived from OLGs provide trophic support to cholinergic neurons.

To test whether anti-BDNF and anti-NT-3 might be additive or synergistic in their effects, we evaluated their combined actions in blockade of CM activity (Fig. 6E). Anti-BDNF added with anti-NT-3 blocked the effects of CM to the same degree as when the factors were added individually. The data support our results with K252a and suggest that OLG-derived factors other than NT-3 and BDNF contribute trophic molecules to cholinergic neurons.
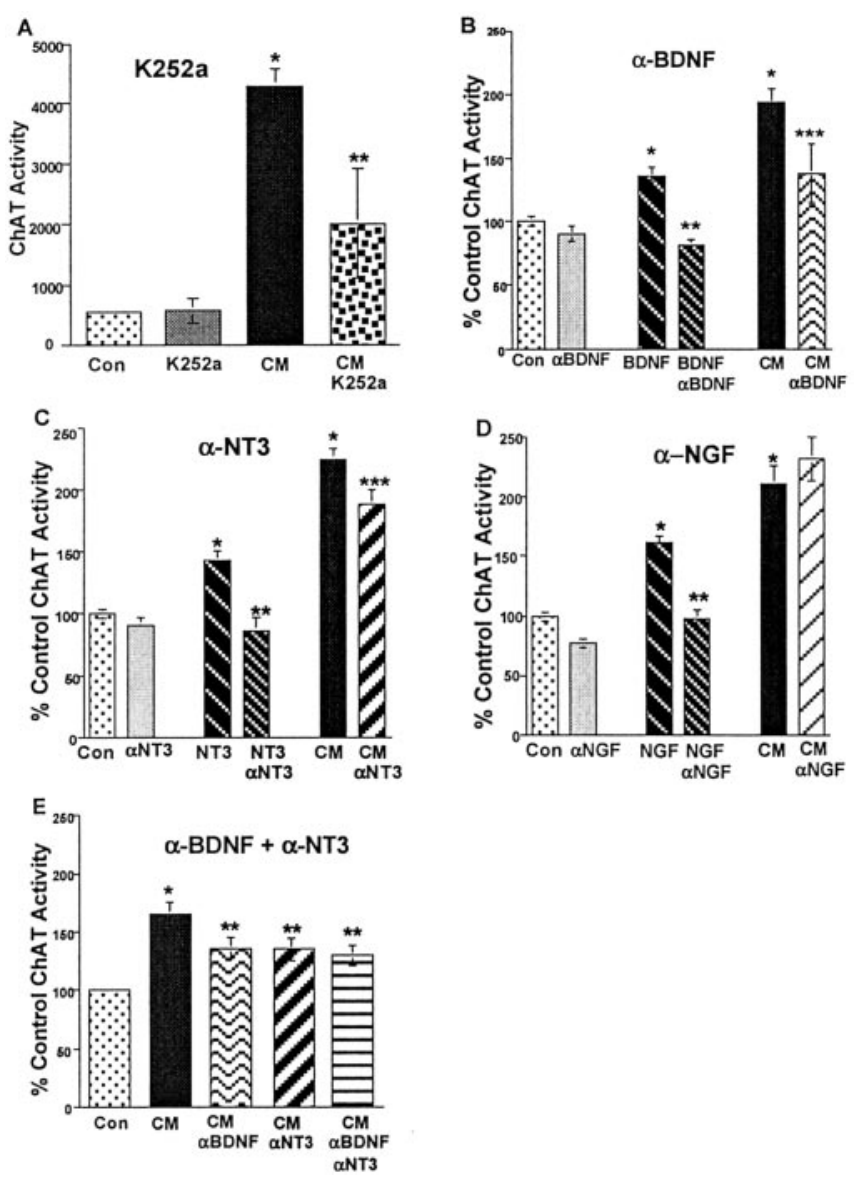

Figure 6. Contribution of neurotrophins to the effects of CM on ChAT activity. A, K252a partially blocks the effects of CM on ChAT activity. One of two independent experiments that yielded similar results are shown. Sample size was $\geq 20$ (5 dishes per group). *Significantly different from control plus DMSO at $p<0.05$. ${ }^{*}$ Significantly different from CM plus DMSO at $p<0.05$. Data were analyzed by ANOVA and the Scheffe's test. $B, C$, Concentrations of antiBDNF ( $\alpha$-BDNF; $B$ ) or anti-NT-3 ( $\alpha$-NT-3; $C$ that completely block the effects of the appropriate neurotrophin partially block the effects of CM on ChAT activity. Combined data from four independent experiments are shown. D, Concentrations of anti-NGF ( $\alpha$-NGF) that completely block the actions of NGF fail to block the effects of CM. Combined data from three independent experiments are shown. $E$, When added together, concentrations of anti-BDNF and anti-NT-3 that block the effects of CM individually have no additional effect on blockade of CM activity. Combined data from three independent experiments are shown. For each neurotrophin tested, sample sizes were as follows: $89(B), 93(C), 72(D)$, and $59(E)$; three or four dishes per group per experiment. *Significantly different from control at $p<0.05{ }^{* *}$ Significantly different from neurotrophin-treated cultures. ${ }^{* *}$ Significantly different from $C M(B, C)$ at $p<0.05$. Data were analyzed by ANOVA and Scheffe's test. Con, Control.

The role of non-OLG lineage cells in the trophic effect

As noted above, our cultures are enriched for OLGs but are not pure. Although the numbers of non-OLG cells vary, generally they make up $<10 \%$ of the total cell number and consist of microglia and astrocytes. Is it possible that these cells are responsible for the trophic influences on neurons observed above? The literature suggests that microglia and astrocytes may play a trophic role. For example, it has been reported that astrocytes (Lindholm al., 1992; Rudge et al., 1992) and microglia (Elkabes et al., 1996) express neurotrophins, which would enhance survival in the BF, and that substantia nigra astrocyte conditioned medium supports survival of substantia nigra neurons (O'Malley et al., 1992). However, conditioned medium from microglia has not been found to provide trophic influences on BF neurons unless these cells are activated (Jonakait et al., 1996). 

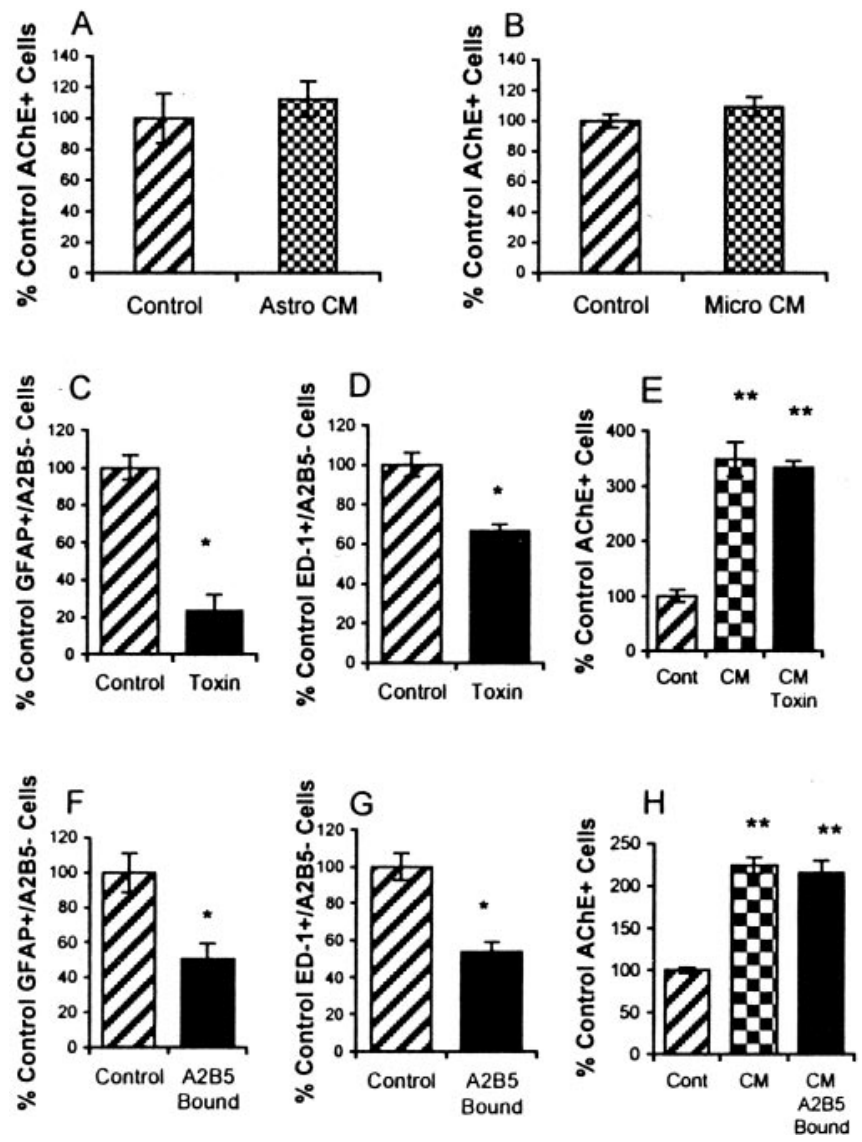

Figure 7. Lack of trophic support by astrocytes and microglia. $A, B$, Numbers of AChEpositive cells are unaffected by astrocyte (Astro) $C M(A)$ or microglia (Micro) $C M(B)$ compared with control serum-free medium. Data represent one of two independent experiments that yielded similar results. Sample size is four $(A)$ or three $(B)$ dishes per group per experiment. $C-E$, Combined treatment with two toxins, $\alpha$-amino adipic acid and leucine methyl ester, significantly reduces numbers of GFAP-positive/A2B5-negative astrocytes ( $C$ and ED-1 microglia (D). However, the effects of $C M$ from toxin-treated cultures (CM Toxin) are not different from effects of $C M$ derived from untreated oligodendrocytes $(C M)(E)$. Data represent one of two experiments that yielded similar results. $\mathrm{F}-\mathrm{H}$, Immunopanning with $\mathrm{A} 2 \mathrm{~B} 5$ antibody (A2B5 Bound) significantly reduces GFAP-positive/A2B5-negative astrocytes ( $F$ ) and ED-1-positive A2B5negative microglia ( $G$ ) compared with untreated cells (Control). However, $C M$ effects from the depleted cultures (CM A2B5 Bound) are not different from CM derived from untreated oligodendrocytes $(C M)$. Data represent one of three experiments that yielded similar results. Sample size was four $(C-E)$ or three $(F-H)$ dishes per group per experiment. *Significantly different from control at $p<0.05$; data were analyzed by Student's $t$ test. ${ }^{*}$ Significantly different from control at $p<0.05$; data were analyzed by ANOVA and Fisher's test. Under control conditions, astrocytes made up $2 \%$ of total cells, and microglia made up $7 \%(\mathrm{C}-\mathrm{H})$.

Therefore, we tested the potential action of the CM derived from contaminating microglia and astrocytes in supporting BF neurons. Initially, we plated astrocytes or microglia in $35 \mathrm{~mm}$ dishes at numbers present in the enriched oligodendrocyte cultures $(10,000 \mathrm{cells} / \mathrm{dish})$. After $4 \mathrm{~d}$, we collected CM, which was tested for trophic effects on neurons. There were none. Neither astrocyte CM nor microglial CM affected the numbers of AChEpositive cells found in the cultures after $7 \mathrm{~d}$ (Fig. 7).

To support this apparent lack of an effect on CM, we used pharmacological ablation and immunopanning approaches to deplete astrocytes and microglia. Cultures were treated with the microglial toxin leucine methyl ester $(10 \mathrm{~mm}, 1 \mathrm{hr})$ and/or the astrocyte toxin $\alpha$-amino adipic acid (600 $\mu \mathrm{M}, 5 \mathrm{~d})$. To monitor the success of the ablation, non-OLG lineage cells were assessed by monitoring ED-1 or OX-42-positive/A2B5-negative microglia or GFAP-positive/A2B5-negative astrocytes. (A2B5 is a marker for oligodendrocyte progenitors. These microglia and astrocytes also did not exhibit myelin basic protein.) When cultures were treated with both toxins, an $\sim 40 \%$ decrease in microglia and an $\sim 80 \%$ decrease in astrocytes were effected. Moreover, trophic effects of the depleted cultures were not reduced compared with controls (Fig. 7). This was true both when contaminating populations were deleted (Fig. 7) and when they were deleted individually (data not shown). To confirm these results, oligodendrocyte progenitors were enriched by immunopanning for A2B5. This procedure resulted in a $50 \%$ decrease in microglia and astrocytes (Fig. 7). Again, significant decreases in microglia and astrocytes did not affect $\mathrm{CM}$ activity.

We cannot rule out the possibility that neurotrophins associated with microglia and astrocytes may contribute to the effects of $\mathrm{CM}$. However, these data, along with our observations that OLGs express neurotrophin mRNA and protein, suggest that neither the microglial nor the astrocyte population plays a dominant role in the trophic actions of the enriched oligodendrocytes.

In sum, our data suggest that BF OLGs express neurotrophins and may provide trophic support to nearby BF neurons. BDNF and NT-3, at least in part, mediate the trophic functions of BF OLGs. In addition, other trophic molecules produced by OLGs maybe also be involved.

\section{Discussion}

Using the BF as a model, our data demonstrate that BF OLGs express neurotrophins in vivo and in culture. We show that OLGs can exert trophic influences on $\mathrm{BF}$ cholinergic neurons by coculturing OLGs with $\mathrm{BF}$ neurons or by treating $\mathrm{BF}$ neurons with OLG CM. In particular, BDNF and NT-3 can mediate the effects of CM on ChAT activity. Our observations suggest an unconventional role of oligodendrocytes as trophin providers for nearby neurons.

\section{Expression of neurotrophins as a characteristic of OLGs}

A variety of glial cells express neurotrophins (Rudge et al., 1992; Zafra et al., 1992). In vivo, astrocytes have been reported to express such trophic molecules, particularly after lesions (Arendt et al., 1995). In addition, microglia in vivo and in culture express neurotrophins (Elkabes et al., 1996). Moreover, these glial cells release molecules that affect nearby neurons. For example, in the CNS, cortical (Jonakait et al., 1996) and BF (Mazzoni and Kenigsberg, 1997) microglial CM enhances differentiation of cholinergic neurons of the BF. Astrocyte $\mathrm{CM}$ of the substantia nigra supports survival of local dopaminergic neurons (O'Malley et al., 1992), whereas astrocytes and OLGs of the superior colliculus produce factors that enhance synaptic activity in retinal ganglion cells (Pfrieger and Barres, 1997). In addition, cortical astrocyte CM supports cortical neuron growth (Le and Esquenazi, 2002). Roles played by neurotrophins in these processes have not been identified. However, it has been noted that NGF, released by depolarizing agents from cortical and hippocampal astrocytes into the medium, increases ChAT activity and numbers of cholinergic neurons in the BF (Abiru et al., 1998) and that BF astrocyte CM also supports survival of cholinergic neurons (our unpublished observation).

Our current work complements and extends these studies, suggesting that OLGs may cooperate with other glial cells to support neurons through the actions of neurotrophins. Previous observations have found that spinal cord oligodendrocytes demonstrate BDNF protein in vivo (Dougherty et al., 2000). Other studies have found that cultured oligodendrocytes from the cortex and hypothalamus express NGF mRNA and protein (Gonza- 
lez et al., 1990; Byravan et al., 1994), and that cultured oligodendrocyte-like cells from the BF express BDNF mRNA and NT-3 mRNA (Dai et al., 2001). We now indicate that NGF, BDNF, and NT-3 mRNAs are expressed not only in OLGs in culture but also in vivo, and this may be a common characteristic of forebrain OLGs. In particular, we show that OLGs in the BF, as well as in the corpus callosum and the frontal and parietal cortices, express NGF, BDNF, and NT-3 mRNAs at P7 in vivo. Thus, forebrain OLGs may serve as a source of neurotrophins for neurotrophin-responsive local neurons or neurons innervating these areas.

Morphologically, oligodendrocytes have been recognized as diverse populations that exhibit distinct distribution patterns (Szuchet, 1995). Our studies suggest that these cells may also be functionally distinct with respect to neurotrophin expression. For example, in situ hybridization examination of the cells in vivo or in culture reveals that no neurotrophin mRNA is expressed in every OLG. Our data suggest that individual oligodendrocytes of the postnatal brain express different neurotrophins and may be distinct from one another. It remains to be determined whether this distinction is maintained throughout life or is confined to the developmental period.

\section{Trophic roles of OLGs}

To directly examine the trophic influences of BF OLGs on nearby neurons, we used OLG-neuron cocultures and OLG CM. The results from these experiments suggest that OLGs support the survival and function of cholinergic neurons, and that this effect is mediated, at least in part, by neurotrophins. In particular, K252a, a tyrosine kinase inhibitor that blocks the signaling cascades for trk neurotrophin receptors also partially inhibits the effect of CM on cholinergic neurons. Moreover, neutralizing antibodies for anti-BDNF and anti-NT-3, but not anti-NGF, partially block the effect of $\mathrm{CM}$ on cholinergic neurons.

\section{Microglia and astrocytes, although capable of expressing neurotrophins, do not play the major role in the CM effect}

The oligodendrocyte cultures have contaminating microglia and astrocytes making up $9 \%$ of the total cell numbers. The literature suggests that these cells may contribute to the action of CM. However, when microglia or astrocytes are plated at numbers present in the enriched oligodendrocyte cultures, they do not express trophic activity. Moreover, when numbers of microglia and astrocytes in OLG cultures are reduced significantly, the trophic effects of the oligodendrocyte cultures are unchanged. These data, along with our observations that oligodendrocytes express both neurotrophin mRNA and protein, suggest that OLGs are the major source of trophic actions.

\section{Other molecules may also play a role}

Importantly, it is worth noting that neurotrophins are not the only trophic molecules that mediate trophic support to nearby cholinergic neurons. As indicated particularly by our experiments using K252a and the experiments that evaluated effects of anti-BDNF together with anti-NT-3, OLG-derived molecules other than neurotrophins may also affect cholinergic neurons. Increasing literature suggests possible candidate trophic molecules. For example, fibroblast growth factor-9 has been reported to be expressed by oligodendrocytes in vivo (Nakamura et al., 1999). Glial cell line-derived neurotrophic factor (GDNF) family members, including GDNF, neurturin, and persepherin, have been detected in OLG cell lines and in primary cells (Strelau and Unsicker, 1999). Insulin-like growth factor-1 (IGF-1) mRNA has been observed in oligodendrocyte progenitors after a short time in culture (Shinar and McMorris, 1995). Neuregulin has been found to be expressed in human white matter and in neonatal oligodendrocytes in culture (Raabe et al., 1997; Cannella et al., 1999). This expression of a broad array of trophic molecules supports the premise that OLGs perform a trophic function in the CNS.

Several additional lines of evidence support our contention that OLGs may play a trophic role and affect the development, survival, and function of neurons. For example, at neonatal stages, unilateral depletion of OLGs in the optic nerve by x-ray exposure significantly reduces the initial increases in axon size that occur during the first 2 weeks of life (Colello et al., 1994). The influence of OLGs on neuronal survival and axonal maintenance is also indicated by axonal swellings and degeneration observed in major proteolipid protein-defective mice (Griffiths et al., 1998). In addition, it has been shown that optic nerve OLGs support long-term survival of retinal ganglion cells by synthesis of as yet unidentified molecules (Meyer-Franke et al., 1995). IGF-1 has been reported recently to be present in cortical OLG $\mathrm{CM}$ and to affect survival of neighboring cortical neurons (Wilkins et al., 2001). Such an effect of OLGs is also suggested by the pathological phenotype of multiple sclerosis (MS), because axonal damage has been revealed by magnetic resonance imaging and immunocytochemistry to be associated with demyelination in MS patients (Ferguson et al., 1997; Trapp et al., 1998).

In summary, we have shown that OLGs can play a trophic function in addition to myelination. This novel role may be important in supporting neurons of the CNS.

\section{References}

Abiru Y, Katoh-Semba R, Nishio C, Hatanaka H (1998) High potassium enhances secretion of neurotrophic factors from cultured astrocytes. Brain Res 809:115-126.

Alderson RF, Alterman AL, Barde YA, Lindsay RM (1990) Brain-derived neurotrophic factor increases survival and differentiated functions of rat septal cholinergic neurons in culture. Neuron 5:297-306.

Arendt T, Bruckner MK, Krell T, Pagliusi S, Kruska L, Heumann R (1995) Degeneration of rat cholinergic basal forebrain neurons and reactive changes in nerve growth factor expression after chronic neurotoxic injury. II. Reactive expression of the nerve growth factor gene in astrocytes. Neuroscience 65:647-659.

Byravan S, Foster LM, Phan T, Verity AN, Campagnoni AT (1994) Murine oligodendroglial cells express nerve growth factor. Proc Natl Acad Sci USA 91:8812-8816.

Cannella B, Pitt D, Marchionni M, Raine CS (1999) Neuregulin and erbB receptor expression in normal and diseased human white matter. J Neuroimmunol 100:233-242.

Colello RJ, Pott U, Schwab ME (1994) The role of oligodendrocytes and myelin on maturation in the developing rat retinofugal pathway. J Neurosci 14:2594-25605.

Dai X, Lercher LD, Yang L, Shen M, Black IB, Dreyfus CF (1997) Expression of neurotrophins by basal forebrain (BF) oligodeudrocytes. Soc Neurosci Abstr 23:331.

Dai X, Vieira C, Lercher LD, Black IB, Dreyfus CF (1998) The trophic role of basal forebrain oligodendrocytes on cholinergic neurons. Soc Neurosci Abstr 24:1778.

Dai X, Qu P, Dreyfus CF (2001) Neuronal signals regulate neurotrophin expression in oligodendrocytes of the basal forebrain. Glia 34:234-239.

DiCicco-Bloom E, Friedman WJ, Black IB (1993) NT-3 stimulates sympathetic neuroblast proliferation by promoting precursor survival. Neuron 11:1101-1111.

Dougherty KD, Dreyfus CF, Black IB (2000) Brain-derived neurotrophic factor in astrocytes, oligodendrocytes and microglial macrophages after spinal cord injury. Neurobiol Dis 7:574-585.

Dreyfus CF, Dai X, Lercher LD, Racey BR, Friedman WJ, Black IB (1999) Expression of neurotrophins in the adult spinal cord in vivo. J Neurosci Res 56:1-7. 
Du Y, Zaremba TM, Lercher LD, Dreyfus CF (2000) p75 influences neurotrophin action on basal forebrain oligodendrocytes. Soc Neurosci Abstr 26:120.17.

Eckenstein F, Soforniew MV (1983) Identification of central cholinergic neurons containing both choline acetyltransferase and acetylcholinesterase and of central neurons containing only acetylcholinesterase. J Neurosci 3:2286-2291.

Elkabes S, DiCicco-Bloom EM, Black IB (1996) Brain microglia/macrophages express neurotrophins that selectively regulate microglial proliferation and function. J Neurosci 16:2508-2521.

Ferguson B, Matyszak MK, Esiri MM, Perry VH (1997) Axonal damage in acute multiple sclerosis lesions. Brain 120:393-399.

Fonnun F (1975) A rapid radiochemical method for the determination of choline acetyltransferase. J Neurochem 24:407-409.

Friedman WJ, Ibanez CF, Hallbook F, Persson H, Cain LD, Dreyfus CF, Black IB (1993) Differential actions of neurotrophins in the locus coeruleus and basal forebrain. Exp Neurol 119:72-78.

Friedman WJ, Black IB, Kaplan DR (1998) Distribution of the neurotrophins brain-derived neurotrophic factor, neurotrophin-3, and neurotrophin-4/5 in the postnatal rat brain: an immunocytochemical study. Neuroscience 84:101-114.

Geneser-Jensen FA, Blackstad TW (1971) Distribution of acetyl cholinesterase in the hippocampal region of the guinea pig. I. Entorhinal area, parasubiculum, and presubiculum. Z Zellforsch Mikrosk Anat 114:460-481.

Gnahn H, Hefti F, Heumann R, Schwab ME, Thoenen H (1983) NGFmediated increase of choline acetyltransferase (ChAT) in the neonatal rat forebrain: evidence for a physiological role of NGF in the brain? Brain Res 285:45-52.

Gonzalez D, Dees WL, Hiney JK, Ojeda SR, Saneto RP (1990) Expression of beta-nerve growth factor in cultured cells derived from the hypothalamus and cerebral cortex. Brain Res 511:249-258.

Griffiths I, Klugmann M, Anderson T, Yool D, Thomson C, Schwab MH, Schneider A, Zimmermann F, McCulloch M, Nadon N, Nave KA (1998) Axonal swellings and degeneration in mice lacking the major proteolipid of myelin. Science 280:1610-1613.

Guillemin G, Boussin FD, Croitoru J, Frank-Duchenne M, Le Grand R, Laszarini F, Dormont D (1997) Obtention and characterization of primary astrocyte and microglial cultures from adult monkey brains. J Neurosci Res 49:576-591.

Hefti F (1986) Nerve growth factor promotes survival of septal cholinergic neurons after fimbrial transections. J Neurosci 6:2155-2162.

Hefti F, Hartikka J, Eckenstein F, Gnahn H, Heumann R, Schwab M (1985) Nerve growth factor increases choline acetyltransferase but not survival or fiber outgrowth of cultured fetal septal cholinergic neurons. Neuroscience 14:55-68.

Jonakait GM, Luskin MB, Wei R, Tian XF, Ni L (1996) Conditioned medium form activated microglia promotes cholinergic differentiation in the basal forebrain in vitro. Dev Biol 177:85-95.

Knusel B, Hefti F (1992) K-252 compounds: modulators of neurotrophin signal transduction. J Neurochem 59:1987-1996.

Knusel B, Michel PP, Schwaber JS, Hefti F (1990) Selective and nonselective stimulation of central cholinergic and dopaminergic development in vitro by nerve growth factor, basic fibroblast growth factor, epidermal growth factor, insulin, and the insulin-like growth factors I and II. J Neurosci 10:558-570.

Le R, Esquenazi S (2002) Astrocytes mediate cerebral cortical neuronal axon and dendritic growth, in part, by release of fibroblast growth factor. Neurol Res 24:81-92.

Martinez HJ, Dreyfus CF, Jonakait GM, Black IB (1985) Nerve growth factor promotes cholinergic development in brain striatal cultures. Proc Natl Acad Sci USA 82:7777-7781.

Mazzoni I, Kenigsberg R (1997) Microglia from the developing rat medial septal area can affect cholinergic and GABAergic neuronal differentiation in vitro. Neuroscience 76:147-157.

McCarthy KD, DeVellis J (1980) Preparation of separate astroglial and oligodendroglial cell cultures from rat cerebral tissue. J Cell Biol 85:890-902.

Meier C, Parmantier E, Brennan A, Mirsky R, Jessen K (1999) Developing
Schwann cells acquire the ability to survive without axons by establishing an autocrine circuit involving insulin-like growth factor, neurotrophin-3, and platelet-derived growth factor-BB. J Neurosci 19:3847-3859.

Meyer-Franke A, Kaplan MR, Pfrieger FW, Barres B (1995) Characterization of the signaling interactions that promote the survival and growth of developing retinal ganglion cells in culture. Neuron 15:805-819.

Mobley WC, Rutkowski JL, Tennekoon GI, Gemski J, Buchanan K, Johnston MV (1986) Nerve growth factor increases choline acetyltransferase activity in developing basal forebrain neurons. Brain Res 387:53-62.

Nakamura S, Todo T, Motoi Y, Haga S, Aizawa T, Ueki A, Ikeda K (1999) Glial expression of fibroblast growth factor-9 in rat central nervous system. Glia 28:53-65.

O’Malley EK, Black IB, Dreyfus CF (1991) Local support cells promote survival of substantia nigra dopaminergic neurons in culture. Exp Neurol 112:40-48.

O’Malley EK, Sieber BA, Black IB, Dreyfus CF (1992) Mesencephalic type I astrocytes mediate the survival of substantia nigra dopaminergic neurons in culture. Brain Res 582:65-70.

O’Malley EK, Sieber BA, Morrison RS, Black IB, Dreyfus CF (1994) Nigral type 1 astrocytes release a soluble factor that increases domaminergic neuron survival through mechanisms distinct from basic fibroblast growth factor. Brain Res 647:83-90.

Pfrieger FW, Barres BA (1997) Synaptic efficacy enhanced by glial cells in vitro. Science 277:1684-1687.

Plant GW, Currier PF, Cuervo EP, Bates ML, Pressman Y, Bunge MB, Wood PM (2002) Purified adult ensheathing glia fail to myelinate axons under culture conditions that enable Schwann cells to form myelin. J Neurosci 22:6083-6091.

Raabe TD, Clive DR, Wen D, DeVries GH (1997) Neonatal oligodendrocytes contain and secrete neuregulins in vitro. J Neurochem 69:1859-1863

Rudge JS, Alderson RF, Pasnikowski E, McClain J, Ip NY, Lindsay RM (1992) Expression of ciliary neurotrophic factor and the neurotrophins nerve growth factor, brain-derived neurotrophic factor and neurotrophin 3 in cultured rat hippocampal astrocytes. Eur J Neurosci 4:459-471.

Sciavolino PJ, Abrams EW, Ynag L, Austenberg LP, Shen MM, Abate-Shen C (1997) Tissue-specific expression of murine Nkx3.1 in the male urogenital system. Dev Dyn 209:127-138.

Shinar Y, McMorris FA (1995) Developing oligodendroglia express mRNA for insulin-like growth factor-I, a regulator of oligodendrocyte development. J Neurosci Res 42:516-527.

Strelau J, Unsicker K (1999) GDNF family members and their receptors: expression and functions in two oligodendroglial cell lines representing distinct stages of oligodendroglial development. Glia 26:291-301.

Szuchet S (1995) The morphology and ultrastructure of oligodendrocytes and their functional implications. In: Neuroglia (Kettenmann H, Ransom BR, eds), pp 23-43. New York: Oxford UP.

Takei N, Tsukui H, Hatanaka H (1988) Nerve growth factor increases the intracellular content of acetylcholine in cultured septal neurons from developing rats. J Neurochem 51:1118-1125.

Toma GJ, Kaplan DR (1997) Neutralization of trkB tyrosine kinase receptor activity using BDNF pAB. Neural Notes 3:20.

Trapp BD, Peterson J, Ransohoff RM, Rudick R, Mork S, Bo L (1998) Axonal transection in the lesions of multiple sclerosis. $\mathrm{N}$ Engl J Med 338:278-285.

Wilkins A, Chandran S, Compston A (2001) A role for oligodendrocytederived IGF-1 in trophic support of cortical neurons. Glia 36:48-57.

Yang L, Zhang H, Hu G, Wang H, Abae-Shen C, Shen MM (1998) An early phase of embryonic Dlx5 expression defines the rostral boundary of the neural plate. J Neurosci 18:8322-8330.

Yokoyama M, Morrison RS, Black IB, Dreyfus CF (1994) Septal neuron cholinergic and GABAergic functions: differential regulation by basic fibroblast growth factor and epidermal growth factor. Brain Res Dev Brain Res 78:201-209.

Zafra F, Lindholm D, Castren E, Hartikka J, Thoenen H (1992) Regulation of brain-derived neurotrophic factor and nerve growth factor mRNA in primary cultures of hippocampal neurons and astrocytes. J Neurosci 12: 4793-4799. 